\title{
Ultralow threshold laser action from toroidal polymer microcavity
}

Abdullah Tulek, Duygu Akbulut, and Mehmet Bayindir

Citation: Appl. Phys. Lett. 94, 203302 (2009);

View online: https://doi.org/10.1063/1.3141730

View Table of Contents: http://aip.scitation.org/toc/apl/94/20

Published by the American Institute of Physics

\section{Articles you may be interested in}

Whispering-gallery mode microdisk lasers

Applied Physics Letters 60, 289 (1998); 10.1063/1.106688

Low-threshold conical microcavity dye lasers

Applied Physics Letters 97, 063304 (2010); 10.1063/1.3479532

Fiber-coupled erbium microlasers on a chip

Applied Physics Letters 83, 825 (2003); 10.1063/1.1598623

Polymer based whispering gallery mode laser for biosensing applications

Applied Physics Letters 106, 031104 (2015); 10.1063/1.4905931

Hybrid microcavity humidity sensor

Applied Physics Letters 102, 241101 (2013); 10.1063/1.4811265

A quasi-droplet optofluidic ring resonator laser using a micro-bubble

Applied Physics Letters 99, 091102 (2011); 10.1063/1.3629814

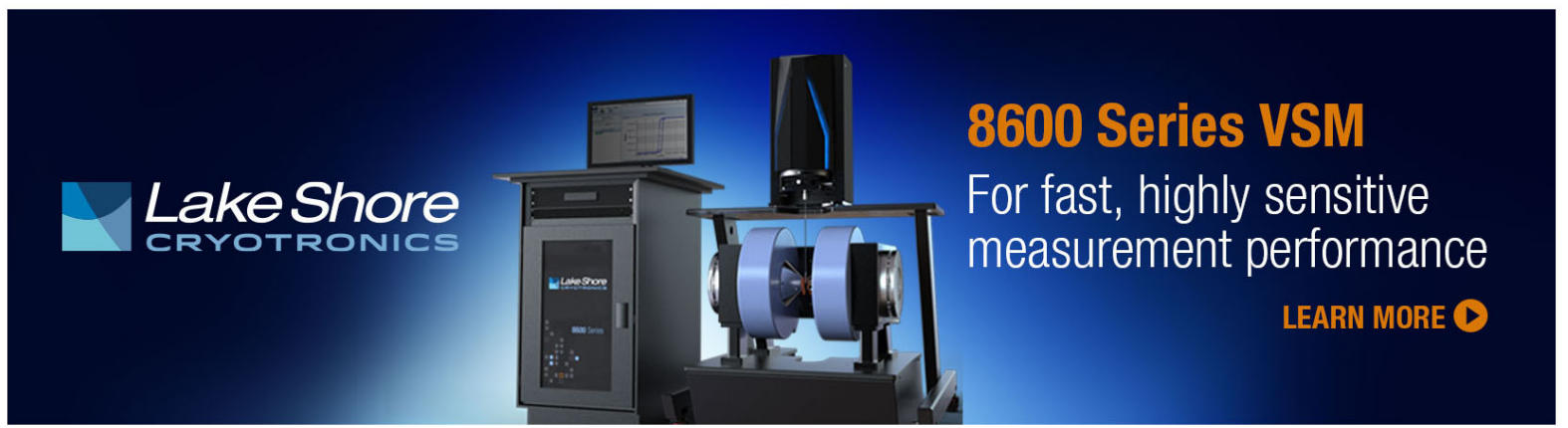




\title{
Ultralow threshold laser action from toroidal polymer microcavity
}

\author{
Abdullah Tulek, ${ }^{1, a)}$ Duygu Akbulut, ${ }^{1}$ and Mehmet Bayindir ${ }^{1,2, b)}$ \\ ${ }^{1}$ UNAM-Institute of Materials Science and Nanotechnology, Bilkent University, 06800 Ankara, Turkey \\ ${ }^{2}$ Department of Physics, Bilkent University, 06800 Ankara, Turkey
}

(Received 9 January 2009; accepted 3 May 2009; published online 21 May 2009)

\begin{abstract}
We report laser action from a toroidal microcavity coated with $\pi$-conjugated polymer. An ultralow threshold value of $\sim 200 \mathrm{pJ} /$ pulse is achieved by free space excitation in ambient conditions. This is the lowest threshold energy obtained in microtoroid lasers by free space excitation. The effective refractive index of the polymer, extracted from Fourier analysis of emission spectra, is 1.787, which is very close to measured value of 1.790 indicating that laser modes are located around the circumference of the cavity as whispering gallery resonances. (C) 2009 American Institute of Physics. [DOI: 10.1063/1.3141730]
\end{abstract}

Microcavities with ultrahigh quality factors have been extensively studied in recent years including the subject of laser action, ${ }^{1,2}$ nonlinear optics, ${ }^{3,4}$ photonics, ${ }^{5}$ quantum information, ${ }^{6}$ optomechanics, ${ }^{7}$ telecommunications, ${ }^{8}$ high resolution spectroscopy, ${ }^{9}$ chemical sensing, ${ }^{10}$ and cavity quantum electrodynamics. ${ }^{11}$ In most of the studies mentioned, microresonators confine light in the form of whispering gallery modes due to very high efficiency of total internal reflection mechanism. Two dimensional photonic crystal structures have also been reported to show ultrahigh quality factors, ${ }^{5}$ which confine light by means of photonic band gap.

Whispering gallery modes are efficient for confining light in a resonator. The requirement for having an ultrahigh quality factor resonator is that the surface roughness of the cavity, where the total internal reflection that occurs has to be much smaller than the operation wavelength, $l \ll \lambda$. Otherwise, as irregularities on the surface increase, the quality factor of the cavity will dramatically decrease due to the scattering of light outside the resonator. ${ }^{12}$ It is not usually possible to obtain such smooth surfaces by employing only lithographic methods and the fabrication of ultrahigh quality factor microcavities necessitates a reflow step where surface tension of the melted structure redefines the shape of resonator. Until now, silica fibers ${ }^{2}$ and microdisks of silica and silicon $^{8,13}$ have been reflowed to generate microspheres and microtoroids, respectively. Microtoroids are usually more advantageous because they can be produced on chip with high quality factors ${ }^{4,13}$ and they can also be engineered to have smaller mode volume, ${ }^{14}$ which is crucial for nonlinear applications. ${ }^{15}$

Laser action from organic gain media with various cavity configurations has been widely studied in a number of contexts due to their ease of fabrication and flexibility in choosing the emission wavelength. ${ }^{16,17}$ Though microspheres with ultrahigh quality factor are widely employed in organic microlasers, ${ }^{16}$ microtoroidal geometry has not yet been combined with organic gain media to demonstrate laser action. In this study we use poly(2,5-dioctyloxy-p-phenylenevinylene) (DOO-PPV), a $\pi$-conjugated polymer, as active medium, which exhibits superior quantum efficiency around $630 \mathrm{~nm}$ (Ref. 20) and observes laser oscillations at very low energy

\footnotetext{
a)Electronic mail: tulek@unam.bilkent.edu.tr.

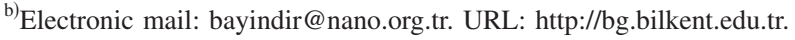

levels with free space excitation. Efficient lasing action is obtained as a result of ultrahigh quality factor of the microstructure and high quantum efficiency of the gain material. Fourier analysis of the emission spectra confirms that resonances are whispering gallery modes and not bow tie.

The essential part of the microlaser fabrication is the creation of silica microtoroids, described in Fig. 1 through its major steps. Although the procedure for microtoroid fabrica- (a)

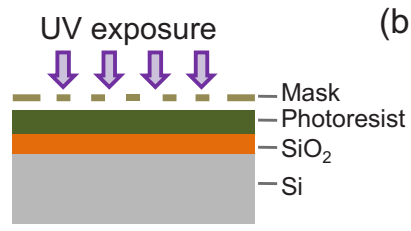

(c) Selective etching of Si

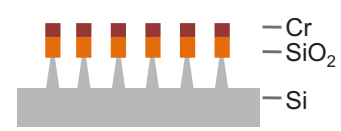

(d)
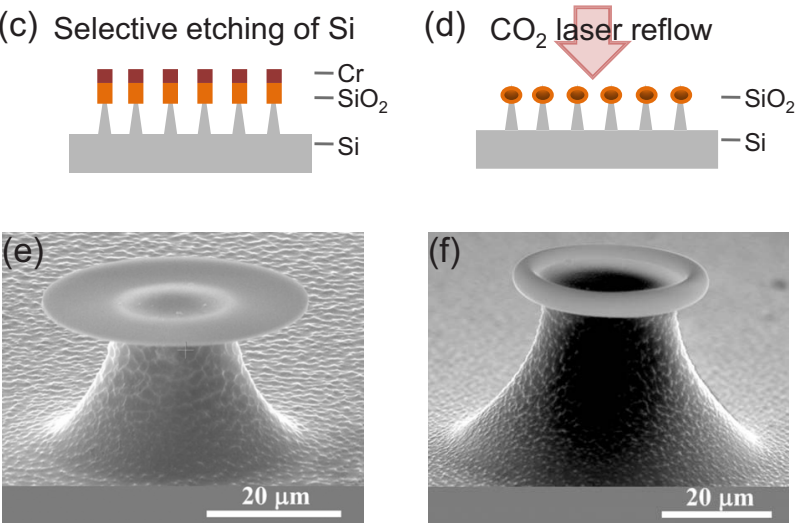

(g)
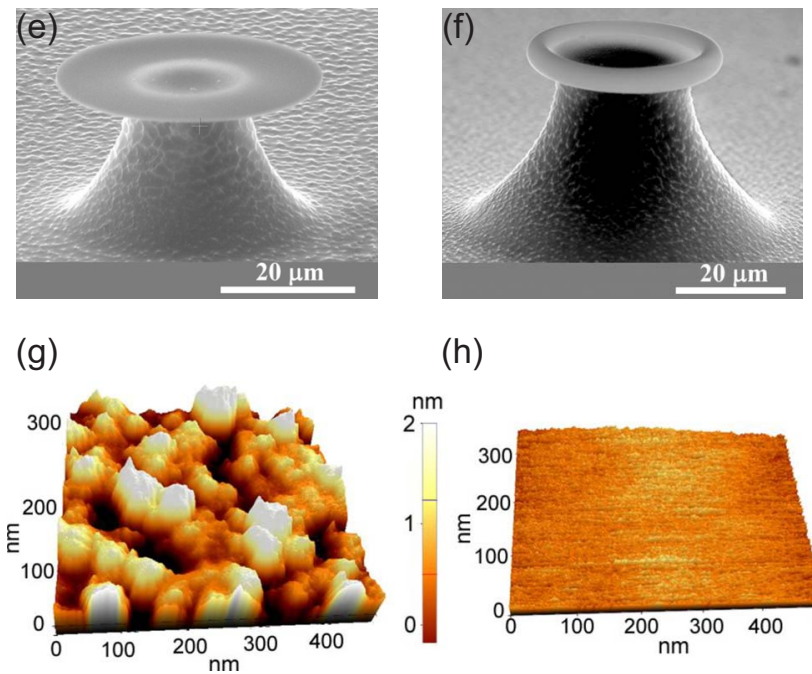

(h)

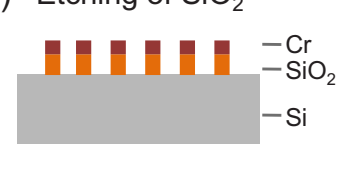

FIG. 1. (Color online) Main steps of the fabrication of silica microtoroids (a) Photolithography of the structure. (b) Transferring microdisks to $\mathrm{SiO}_{2}$ by dry etching. (c) Selective etching of silicon. (d) Reflowing the undercut disks with $\mathrm{CO}_{2}$ laser. (e) The undercut disk after $\mathrm{Si}$ is etched. (f) The reflowed microdisk attaining toroidal shape. AFM images of $(\mathrm{g})$ the disk and (h) toroid surface. 


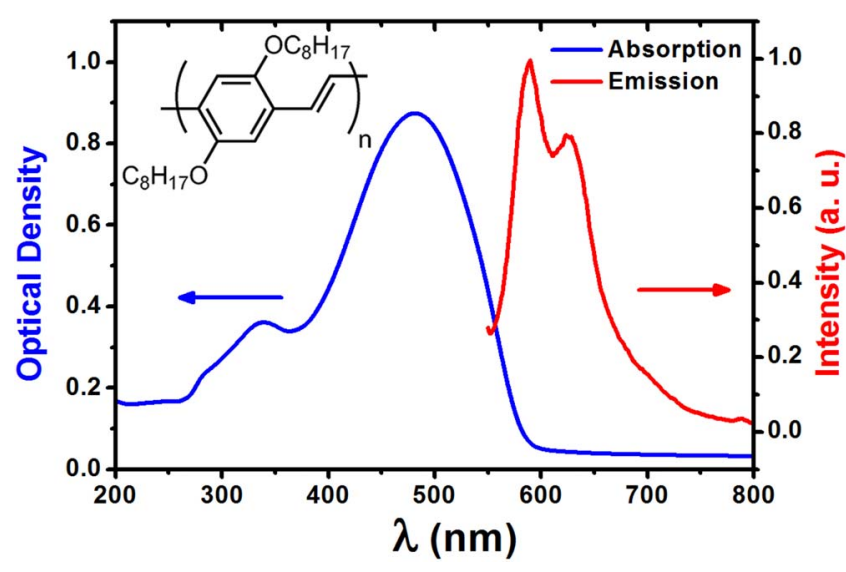

FIG. 2. (Color online) Absorption and emission spectrum of $\sim 1 \mu \mathrm{m}$ thick DOO-PPV polymer film. Emission is taken at an excitation wavelength of $520 \mathrm{~nm}$. The inset is the chemical structure of DOO-PPV polymer.

tion has been previously reported, ${ }^{13}$ the details of our process are different and therefore explained here. A positive photoresist is spun on top of a thermally grown $\sim 1 \mu \mathrm{m}$ thick $\mathrm{SiO}_{2}$ layer on a low doped silicon substrate. After soft baking at $110{ }^{\circ} \mathrm{C}$ for $1 \mathrm{~min}$, the resist is exposed to UV light in the presence of a chromium mask for defining disk structures on the resist material. Next the resist is postbaked at $110^{\circ} \mathrm{C}$ for $2 \mathrm{~min}$ and flood exposed to UV light again for generating the reverse of the image on the mask [Fig. 1(a)]. This technique produces disk shaped vacancies in the substrate. After developing, the vacancies are then filled with $\sim 100 \mathrm{~nm}$ thick $\mathrm{Cr}$ layer. Subsequently the whole ensemble is exposed to a lift off process leaving only $\mathrm{Cr}$ disk structures on top of the oxide layer. Once the disks are obtained, they are transferred to the $\mathrm{SiO}_{2}$ layer via anisotropic etching with the plasma of the $\mathrm{CHF}_{3}$ and $\mathrm{O}_{2}$ mixture [Fig. 1(b)]. The isotropic selective etching of silicon is accomplished with the plasma of $\mathrm{SF}_{6}$ yielding $\mathrm{SiO}_{2}$ microdisks on top of $\mathrm{Si}$ pillars [Figs. 1(c) and $1(\mathrm{e})]$.

Disk microcavities are exposed to a $\mathrm{CO}_{2}$ laser with $280 \mathrm{~W} / \mathrm{mm}^{2}$ intensity for $3 \mathrm{~min}$ reflowing the structure to produce the final smooth toroidal microcavity via surface tension [Figs. 1(d) and 1(f)]. The $Q$-factor of fabricated microtoroids is not measured, however, it has been previously reported numerous times that this quantity is between $10^{7}$ and $10^{8}$ for this configuration of material and geometry. $1,10,13$ To characterize the effect of surface tension induced changes on microstructures better, surface roughness of cavities is measured before and after the reflow process via noncontact atomic force microscopy (AFM) (Park Scientific, XE-100). The surface roughness of the microdisks before reflow is measured as $1.2 \mathrm{~nm}$, whereas after reflow it is reduced to 0.2 $\mathrm{nm}$ on the rim of the microtoroid [Figs. 1(g) and 1(h)], explaining substantial improvement on the quality factor. ${ }^{13}$

Following the fabrication of the silica microtoroids, DOO-PPV, organic gain medium emitting in the red region (Fig. 2), is dissolved in toluene at $10 \mathrm{mg} / \mathrm{ml}$ concentration and spun onto the structures at $1000 \mathrm{rpm}$ yielding an approximate thickness of $1.5 \mu \mathrm{m}$ on the outer surface of the resonators. A subsequent baking of the whole ensemble at $90{ }^{\circ} \mathrm{C}$ in rough vacuum for $6 \mathrm{~h}$ ensures both cleanliness and smoothness of the polymer covering the toroid surface [Fig. $3(\mathrm{a})$ ]. It is determined from AFM measurements that the surface roughness of polymer coated microtoroid is degraded to
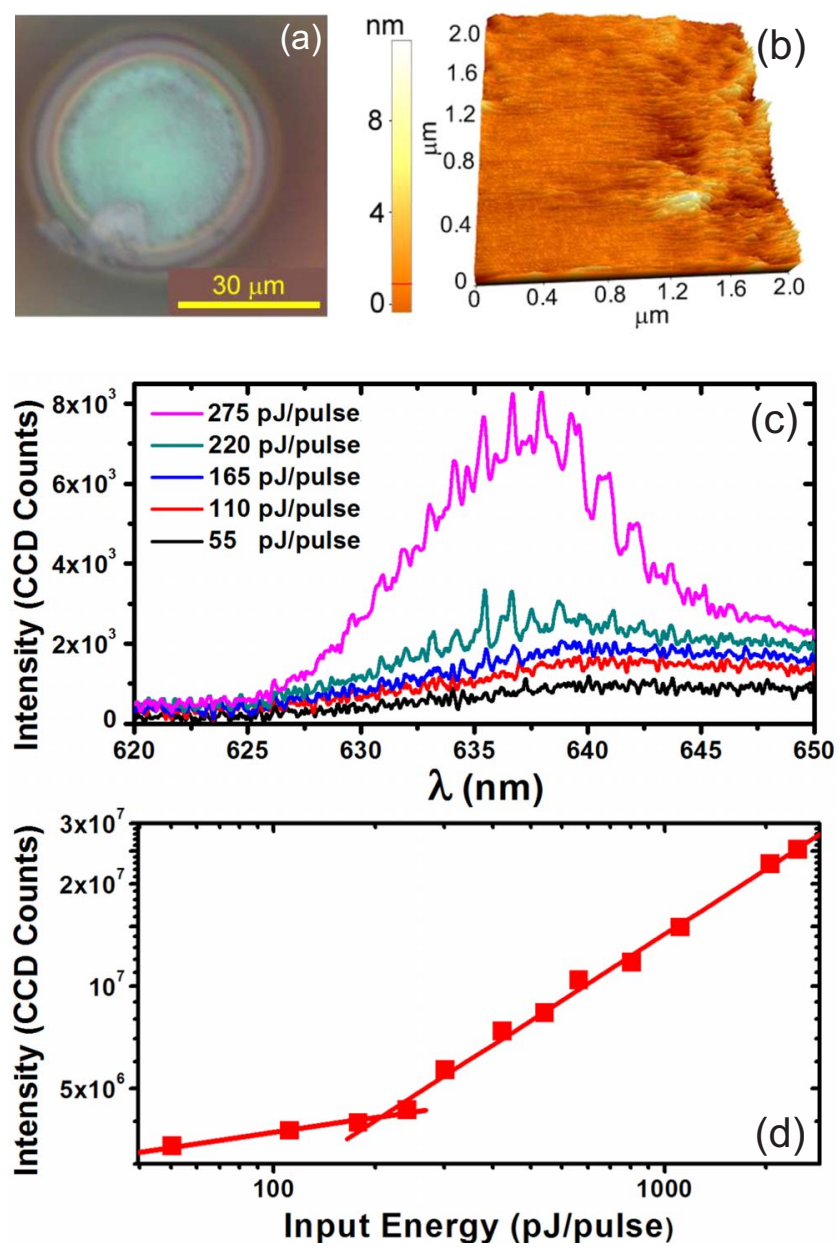

FIG. 3. (Color online) (a) Optical and (b) AFM image of the polymer coated microtoroid. The inner and outer diameters of the toroid are 44 and $56 \mu \mathrm{m}$, respectively. Note that the DOO-PPV thin film (light orange color) covers the whole structure. (c) Evolution of the microtoroid laser emission spectrum with respect to excitation energy level. (d) Integrated emission intensity vs excitation energy level exhibiting a kink at threshold energy of 200 $\mathrm{pJ} / \mathrm{pulse}$.

\section{$1.3 \mathrm{~nm}$ with respect to the silica sample [Fig. 3(b)].}

To observe lasing action, polymer microtoroids are optically excited by a femtosecond parametric amplifier system (Spectra Physics) operating in sum frequency generation mode at $521.6 \mathrm{~nm}$ wavelength with a pulse duration of $130 \mathrm{fs}$ and repetition rate of $1 \mathrm{kHz}$. Light is focused loosely to each microcavity from the top with a lens where the effective excitation area is $2.5 \mathrm{~mm}^{2}$. Laser emission from polymer microtoroids is collected via an optical fiber and sent to a charge coupled device coupled $1 / 2 \mathrm{~m}$ triple spectrometer where band pass resolution is $0.14 \mathrm{~nm}$ (Spectral Products).

Excitation intensity upon the polymer microtoroids is changed via a variable neutral density filter where, at low energies, only a weak amplified spontaneous emission (ASE) signal is observed without any discrete laser modes. However, as the intensity increases, narrow laser emission peaks with $0.25 \mathrm{~nm}$ full width half maximum starts to emerge in the recorded spectra [Fig. 3(c)]. The threshold of excitation energy level where laser peaks starts to occur is $\sim 170 \mathrm{pJ} /$ pulse. As the excitation power is raised further, the intensity of laser lines continues to increase as well as the ASE becomes more pronounced. We do not anticipate a particular change in the laser emission spectrum as the pulse duration gets longer to picosecond time scales. ${ }^{18}$ However its 


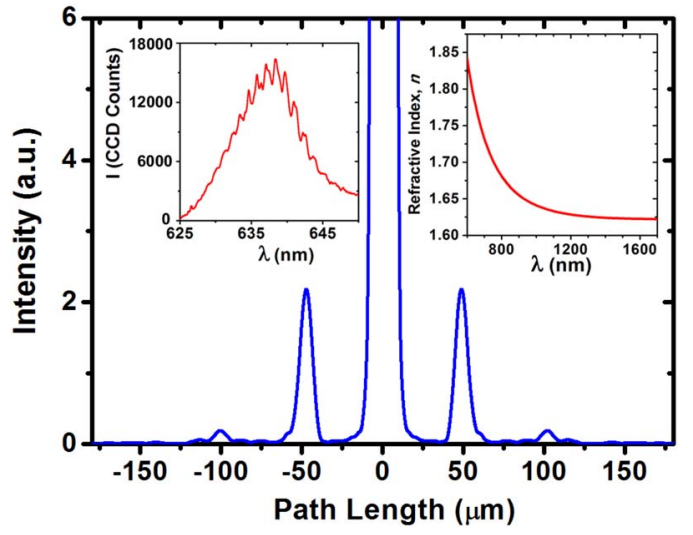

FIG. 4. (Color online) Power Fourier transform of the laser emission spectrum shown in the left inset taken at the energy level of $440 \mathrm{pJ} / \mathrm{pulse}$. Right inset shows the ellipsometric refractive index measurement of the DOOPPV polymer.

dynamic behavior can be different due to the possibility of multiple excitations for pulse durations longer than the ASE lifetime. ${ }^{19}$ The thermal effects become more pronounced when the excitation pulse is in nanosecond time scale, resulting in a slight broadening of the emission spectrum. ${ }^{20}$

The laser threshold can be better determined using bilinear behavior of the integrated emission intensity collected from toroid microlaser as a function of excitation energy level. As can be seen from Fig. 3(d), the output intensity of the microlaser has an apparent kink in its dependence to excitation energy at $\sim 200 \mathrm{pJ} /$ pulse. The threshold for the microtoroid laser is one order of magnitude smaller than the previous studies, ${ }^{1}$ confirming the high efficiency of the gain medium.

Spectral separation of laser lines of microresonators where light is confined in the form of whispering gallery resonances is expressed by ${ }^{17,18} \Delta \lambda=\lambda^{2} / n C$, where $\lambda$ is the emission wavelength, $n$ is the effective refractive index of the gain medium, and $C$ is the microresonator circumference. The relation presumes that the microresonator is a FabryPérot type cavity; therefore Fourier transform of the emission spectrum contains equally spaced diminishing Fourier components with periodicity $\Delta l=n C / 2 \pi$. Such an approximation is commonly used in the literature for microresonators where modes are located nearby the circumference of cavity. ${ }^{18}$ The Fourier transform of the emission spectrum (Fig. 4, left inset) for our microtoroid laser is shown in Fig. 4 , where the $x$-axis shows the optical path length to facilitate calculation of $n$. From the previous relation, we can deduce spacing between Fourier components for toroidal geometry as $\Delta l=n D / 2$, where $D$ is the major diameter of the microtoroid. The transform data give $\Delta l=50.5 \mu \mathrm{m}$ and by using microtoroid laser diameter $D=56.5 \pm 0.5 \mu \mathrm{m}$. The effective refractive index $n$ of the polymer is found to be 1.787, which is very close to its measured value of 1.790 acquired at $637 \mathrm{~nm}$ wavelength (Fig. 4, right inset). This result indicates that whispering gallery resonances are mostly located in the polymer medium rather than the $\mathrm{SiO}_{2}$ template. Consequently they are located around the circumference of the microcavity, which is enforced by total internal reflection. ${ }^{18}$ It is also important to note that the lasing action takes place on the outer surface of the microtoroid, because the thickness of the gain medium around the inner circle of toroid is much less than the outer.

In conclusion, we demonstrated laser action from a polymer coated microtoroid with substantially low threshold energy for free space excitation. It is shown that lowering the threshold energy of a microlaser is possible by using superior confinement properties of microtoroids and high quantum efficiency of an organic gain media. Lowering the laser threshold energy in a free space excitation system can be important to avoid difficulties related to coupling of excitation light with a tapered fiber. Furthermore the Fourier analysis of the emission spectra verified that laser modes are mostly located around the periphery of the microcavity as whispering gallery resonances.

This work is supported by TUBITAK under the Project No. 106T348 and 106G090. M.B. acknowledges support from the Turkish Academy of Sciences Distinguished Young Scientist Award (TUBA GEBIP). This work was performed at the UNAM-Institute of Materials Science and Nanotechnology supported by the State Planning Organization of Turkey through the National Nanotechnology Research Center Project. We thank Professor Z. V. Vardeny at the University of Utah for providing the DOO-PPV powder used in our experiments.

${ }^{1}$ B. Min, S. Kim, K. Okamoto, L. Yang, A. Scherer, H. Atwater, and K. Vahala, Appl. Phys. Lett. 89, 191124 (2006).

${ }^{2}$ V. Sandoghdar, F. Treussart, J. Hare, V. Lefevre-Seguin, J. M. Raimond, and S. Haroche, Phys. Rev. A 54, R1777 (1996).

${ }^{3}$ P. Del'Haye, A. Schliesser, O. Arcizet, T. Wilken, R. Holzwarth, and T. J. Kippenberg, Nature (London) 450, 1214 (2007).

${ }^{4}$ S. M. Spillane, T. J. Kippenberg, and K. J. Vahala, Nature (London) 415 , 621 (2002).

${ }^{5}$ B.-S. Song, S. Noda, T. Asano, and Y. Akahane, Nature Mater. 4, 207 (2005).

${ }^{6}$ P. Bianucci, C. R. Fietz, J. W. Robertson, G. Shvets, and C.-K. Shih, Phys. Rev. A 77, 053816 (2008).

${ }^{7}$ T. Carmon and K. J. Vahala, Phys. Rev. Lett. 98, 123901 (2007).

${ }^{8}$ J. Yao, D. Leuenberger, M.-C. M. Lee, and M. C. Wu, IEEE J. Quantum Electron. 13, 202 (2007).

${ }^{9}$ G. Schweiger, R. Nett, and T. Weigel, Opt. Lett. 32, 2644 (2007).

${ }^{10}$ A. M. Armani and K. J. Vahala, Opt. Lett. 31, 1896 (2006).

${ }^{11}$ T. Aoki, B. Dayan, E. Wilcut, W. P. Bowen, A. S. Parkins, T. J. Kippenberg, K. J. Vahala, and H. J. Kimble, Nature (London) 443, 671 (2006).

${ }^{12}$ A. I. Rahachou and I. V. Zozoulenko, Appl. Opt. 43, 1761 (2004).

${ }^{13}$ D. K. Armani, T. J. Kippenberg, S. M. Spillane, and K. J. Vahala, Nature (London) 421, 925 (2003).

${ }^{14}$ S. M. Spillane, T. J. Kippenberg, K. J. Vahala, K. W. Goh, E. Wilcut, and H. J. Kimble, Phys. Rev. A 71, 013817 (2005).

${ }^{15}$ D. Akbulut, A. Tulek, and M. Bayindir, in Proceedings of 2008 10th Anniversary International Conference on Transparent Optical Networks, edited by M. Marciniak (IEEE, New York, 2008), Vol. 4, pp. 260-263.

${ }^{16}$ M. Tanyeri, R. Perron, and I. M. Kennedy, Opt. Lett. 32, 2529 (2007).

${ }^{17}$ A. Tulek and Z. V. Vardeny, Appl. Phys. Lett. 91, 121102 (2007).

${ }^{18}$ R. C. Polson, G. Levina, and Z. V. Vardeny, Appl. Phys. Lett. 76, 3858 (2000).

${ }^{19}$ J. D. Huang, S. Frolov, Z. Vardeny, C. W. Lee, and K. S. Wong, Synth. Met. 116, 61 (2001).

${ }^{20}$ S. V. Frolov, M. Shkunov, A. Fujii, K. Yoshino, and Z. V. Vardeny, IEEE J. Quantum Electron. 36, 2 (2000). 